\title{
TAXA DE DOSE ERITEMATOSA SOB CÉU ENCOBERTO POR ALTO-ESTRATO: ESTUDO DE CASO
}

\author{
Abel A. Silva \\ Recebido em 10 novembro, 2009 / Aceito em 17 junho, 2010 \\ Received on November 10, 2009 / Accepted on June 17, 2010
}

ABSTRACT. The erythemal dose rate (EDR) on the Earth's surface depends mainly on the solar zenith angle, column ozone, clouds, aerosols and surface albedo. Clouds are the main atmospheric agent in the modulation of EDR. Therefore, an investigation into the effect of clouds on the ultraviolet radiation (UVR) incidence is an important matter due to the effects of UVR on the biosphere. In this work, a case study is reported where EDR incidences are presented for an overcast-sky scenario of Altostratus (As) in Belo Horizonte (BH, $19.92^{\circ} \mathrm{S}, 43.94^{\circ}$ 0, $858 \mathrm{~m}, 331 \mathrm{~km}^{2}$, Brazil). Although the attenuation yielded by the As layer, the incidence of UVR remained high setting in a threatening weather condition for people in outdoor activities.

Keywords: solar ultraviolet radiation, clouds, cloud cover, UV-Index.

RESUMO. A taxa de dose eritematosa (TDE) na superfície terrestre devido à radiação ultravioleta (RUV) emitida pelo Sol depende principalmente do ângulo solar de zênite, da coluna de ozônio, da cobertura de nuvens, dos aerossóis em suspensão e do albedo de superfície. As nuvens são o mais importante agente atmosférico na modulação da TDE, e uma investigação detalhada do efeito produzido por elas na incidência de RUV se justifica pelo impacto dessa radiação na biosfera. Neste trabalho, descreve-se um estudo de caso para a incidência de TDE sob céu completamente encoberto por alto-estrato (As) em Belo Horizonte $\left(\mathrm{BH}, 19,92^{\circ} \mathrm{S}, 43,94^{\circ} \mathrm{O}\right.$, $858 \mathrm{~m}, 331$ km² , Brasil). Apesar da RUV ser atenuada pela camada de As, isso não é suficiente para reduzir a incidência da mesma em termos de TDE a níveis seguros, representando uma ameaça às pessoas em atividades ao ar livre.

Palavras-chave: radiação solar ultravioleta, nuvens, cobertura de nuvens, Índice UV. 


\section{INTRODUÇÃo}

A radiação ultravioleta (RUV) emitida pelo Sol afeta a biosfera terrestre de várias formas. Em relação aos seres humanos, ela pode trazer benefícios como a síntese de vitamina $\mathrm{D}$ ou malefícios como 0 eritema, 0 bronzeado, 0 câncer de pele, 0 enveIhecimento precoce da pele, a imunossupressão e etc (Kullavanijaya \& Lim, 2005; Peters et al., 2009). A intensidade de RUV na superfície terrestre é em função principalmente do ângulo solar de zênite (ASZ), da coluna de ozônio, das nuvens, dos aerossóis e do albedo de superfície. Apesar da ameaça de mudanças globais devido à ação antropogênica (McKenzie et al., 2007), 0 crescimento mundial do número de casos de câncer de pele se deve muito mais ao estilo de vida humano em relação ao Sol do que a qualquer mudança já identificada - a redução da camada de 0zônio, por exemplo - (Godar, 2005; Silva, 2007, 2009). Embasando essa tese estão algumas interpretações erradas da condição meteorológica diária como, por exemplo, a de que as nuvens sempre atenuam, e a níveis seguros, a incidência de RUV (WHO, 2002; Piacentini et al., 2003).

As nuvens são 0 mais importante agente atmosférico na modulação de RUV solar (Borkowski et al., 1977; McKenzie et al., 2007). A RUV incidente no solo pode ser atenuada na passagem através das nuvens ou amplificada por reflexão em certos tipos de nuvens. Ambas as situações podem ocorrer numa questão de minutos (Schafer et al., 1996; Mayer et al., 1998; Cede et al., 2002; Crawford et al., 2003; Krzyscin et al., 2003; Piacentini et al., 2003; Lovengreen et al., 2005; Sabburg \& Parisi, 2006; Adam \& Shazly, 2007). Medidas de cobertura de nuvens por observações visuais em solo são uma antiga prática meteorológica sujeita às inevitáveis tendências e incertezas produzidas pelo olho e interpretação humanos. Nas últimas décadas, a observação da cobertura de nuvens a partir de satélites em órbita e equipamentos automatizados em solo têm incrementado a aquisição de dados sobre nuvens (Holle \& MacKay, 1975; Herman et al., 2001a,b; Schoeberl et al., 2004; Long et al., 2006; Souza-Echer et al., 2006). Contudo, o efeito de uma nuvem sobre a RUV depende de sua forma, altura, posição e composição, fazendo com que a descrição do efeito tenha uma incerteza elevada, mesmo para um céu completamente encoberto (Calbó et al., 2005).

Neste trabalho, desenvolvido numa capital brasileira representante de grande centro urbano, um estudo de caso é relatado onde a taxa de dose eritematosa (TDE) incidente atinge níveis altos, a despeito da predominância de céu completamente encoberto por uma camada de alto-estrato (As) ao longo do dia. Tal cenário representa uma ameaça às pessoas em atividade ao ar livre em termos de exposição excessiva à RUV, num cenário em que aparentemente a incidência de radiação é reduzida pela camada de nuvens. Estudos já mostraram que a população brasileira se expõe em excesso à radiação solar (Benvenuto-Andrade et al., 2005; Szklo et al., 2007), o que se deve em geral à desinformação ou descrença nos potenciais efeitos danosos da RUV sobre a saúde (Souza et al., 2004). 0 câncer de pele é o tipo mais comum de câncer no Brasil (INCA, 2007), e muito provavelmente esse fato se deve aos hábitos da população em relação ao Sol. Tais hábitos poderiam ser mitigados com mais informação sobre os benefícios e malefícios da exposição à RUV solar (Silva, 2008; Peters et al., 2009) inclusive em dias nublados. Embora este trabalho relate apenas um caso referente à cobertura de As, ele mostra uma situação bastante comum nos trópicos: a exposição diária a altas intensidades de RUV solar mesmo sob uma cobertura de nuvens. Portanto, um dos objetivos deste trabalho é aumentar o conhecimento relativo à exposição solar sob condições de nebulosidade, para que essa informação esteja disponível em campanhas de conscientização do público em geral sobre a exposição à radiação solar, mostrando de forma quantitativa que mesmo num cenário de céu encoberto ainda pode haver um risco elevado de dano à saúde devido à RUV solar.

\section{MATERIAL E MÉTODOS}

0 Laboratório de Luz Ultravioleta (LLUV, www.dfq.pucminas.br/ PUV/index.html) está sediado em Belo Horizonte (BH, 19,92 ${ }^{\circ} \mathrm{S}$, 43,94ㅇ, $858 \mathrm{~m}, 331 \mathrm{~km}^{2}$, Brasil), uma cidade com clima tropical de altitude, no centro da terceira maior região metropolitana do país e cuja economia é baseada na atividade industrial e no comércio, representando uma fonte significativa de poluentes para a atmosfera. Um Biômetro Solar Light 501A (BSL, Solar Light Co., Inc., Glenside, USA) e um imageador Total Sky Imager 440A (TSI, Yankee Environmental Systems, Inc., Turners Falls, USA) foram instalados na torre do LLUV dentro do campus da Pontifícia Universidade Católica de Minas Gerais (PUC Minas) (Silva, 2009).

0 BSL mede a RUV solar ponderada pelo efeito de eritema através de uma curva-resposta similar à curva obtida no trabaIho de McKinlay \& Diffey (1987). Ele é calibrado nas instalações do fabricante a cada 12 meses de uso, além de suas medidas serem corrigidas para a resposta-cosseno e sua base de tempo ser mantida dentro de uma incerteza de 10 segundos. A incerteza na medida de dose eritematosa (DE, que é a intensidade de RUV ponderada pelo espectro de ação para 0 efeito de eritema integrada num dado intervalo de tempo (WHO, 2002)) 
depende dos erros aleatório, de calibração e de correção à resposta-cosseno do sensor do BSL, sendo 1,6\%, 6,2\% (conforme certificado de calibração) e 2,5\%, respectivamente, os valores para essas incertezas (Hülsen \& Gröbner, 2007). Portanto, a incerteza total numa medida de DE é 6,9\%. As medidas são armazenadas no registrador do BSL e podem ser acessadas por computador através de programas para conexão via terminais. A taxa de dose eritematosa (TDE) é obtida da razão entre a DE e 0 tempo gasto na medida (6 minutos neste caso). Assim, na verdade, a medida de TDE é um valor médio.

0 TSI mede a cobertura de nuvens local (fração de céu encoberto por nuvens vista a partir de um ponto no solo) e pode ser descrito como sendo uma câmara CCD (Charged Coupled Device) direcionada para baixo, posicionada acima de um espelho esférico cuja face refletora está voltada para o céu (ou seja, para a câmara). Sobre o espelho existe uma tira negra que impede a reflexão direta da radiação solar na CCD. A câmara fotografa (1 foto/minuto neste caso) imagens de céu refletidas no espelho. A imagem é armazenada no computador onde o programa de gerenciamento do TSI controla a operação do mesmo. Posteriormente, essas imagens são tratadas por uma função dentro desse mesmo programa, mensurando qual é a razão entre os montantes das cores vermelho e azul em cada pixel da imagem. 0 valor dessa razão define se há uma nuvem ou não naquela parte da imagem (Pfister et al., 2003). Em geral, a existência de uma nuvem produz setores onde a presença da cor vermelha é maior que a da cor azul. Contudo, problemas de identificação do que é e do que não é nuvem numa imagem são comuns, mesmo depois de obtido um ajuste adequado dos parâmetros que definem essa função no programa de gerenciamento do TSI: aerossóis, por exemplo, podem ser confundidos com nuvens. A base de tempo do TSI também é mantida dentro de uma incerteza de 10 segundos. No processamento das imagens para determinação da cobertura de nuvens restringe-se 0 ângulo de abertura das mesmas a $100^{\circ}$ (50 em torno do zênite), o que elimina dois problemas: (i) a distorção da imagem pela curvatura do espelho e (ii) a inevitável falsa impressão de incremento da cobertura de nuvens devido ao efeito de visão em perspectiva das regiões da abóbada celeste com ângulos de zênite maiores.

Assim, esses dois equipamentos trabalharam simultaneamente em $\mathrm{BH}$, realizando medidas de cobertura de nuvens e de incidência de TDE para uma grande variedade de cenários de céu. Alguns desses cenários chamam a atenção pelo risco que eles trazem à saúde de pessoas em atividades ao ar livre devido a valores elevados de TDE incidente, ainda que sob 100\% de cobertura de nuvens como ocorreu no dia 21 de outubro de 2007.

\section{RESULTADOS E DISCUSSÃO}

0 dia 21 de outubro de 2007, dia 294 do ano, foi um dia com predominância de nuvens de média e alta altitude tais como altoestrato (As) e cirro (Ci) (WHO, 1956). Em particular, uma espessa camada de As esteve sobre BH de 13:30 às 15:24 hora universal (HU, de 11:30 às 13:24 horário local de verão), produzindo um céu completamente encoberto cujas imagens foram registradas pelo TSI, enquanto o BSL media a TDE incidente. Esse cenário de cobertura de nuvens associado (i) a uma temperatura amena típica da estação (temperatura típica em torno dos $25^{\circ} \mathrm{C}$ ), (ii) à ausência de chuva e (iii) ao fato de ser domingo, um dia devotado ao lazer, muito provavelmente serviu de estimulo às pessoas para a prática de atividades ao ar livre. Os estudos conduzidos por Benvenuto-Andrade et al. (2005) e Szklo et al. (2007) mostram que no Brasil há uma tendência, principalmente entre os mais jovens, de se expor mais ao Sol e adotar menos as medidas de proteção numa exposição, especialmente durante a prática de atividades físicas ao ar livre. Por outro lado, 0 fato de a camada de As ser espessa o bastante para impossibilitar a formação de sombra dos corpos, mas fina o suficiente para produzir um nível de iluminação confortável aos olhos pode ter se somado à tendência observada por aqueles autores para que muitas pessoas, em especial jovens, tenham pressuposto que a incidência de RUV solar fosse baixa, não havendo razão para se preocuparem com ela. No entanto, se o dia está nublado não significa que a incidência de RUV é baixa, mesmo porque as nuvens apresentam maior transparência à RUV do que à luz visível (Seckmeyer et al., 1996; Kylling et al., 1997).

A análise visual das fotos no período citado acima (Fig. 1) mostra claramente que havia pelo menos uma espessa camada de As encobrindo todo o céu. É possível que uma camada de Ci também estivesse presente acima da camada de As, uma vez que um halo (efeito ótico produzido pela incidência de luz solar sobre uma camada de cristais de gelo em suspensão na atmosfera (WMO, 1956)), acusando a presença de uma nuvem $\mathrm{Ci}$, foi observado nas imagens do TSI no período da manhã. A parte superior da Figura 2 mostra os valores de cobertura de nuvens medidos pelo TSI para aquele dia, sendo esses valores médias corridas em intervalos de 15 minutos tomados a cada 1 minuto. A cobertura de nuvens obtida pelo processamento das imagens do TSI foi superior a $90 \%$ durante a maior parte do período diurno. Na parte inferior da Figura 2 são vistos os valores medidos da TDE incidente, além das faixas de Índice UV (IUV, número inteiro a partir de zero resultante do arredondamento do cociente da divisão da TDE por 0,025 W/m²) à direita (WHO, 2002; Silva, 

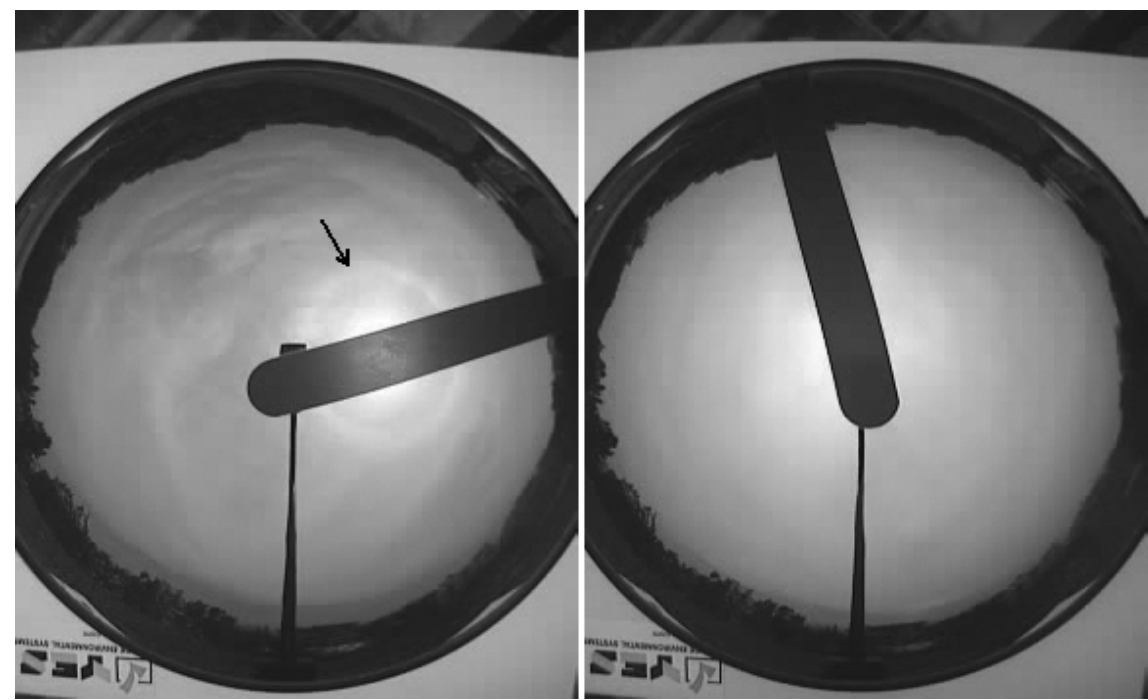

Figura 1 - Imagens do TSI em BH no dia 21 de outubro de 2007: (Esquerda) às 13:00 HU mostrando a cobertura de Ci e 0 halo indicado pela seta; (Direita) às 14:50 HU mostrando a cobertura de As.
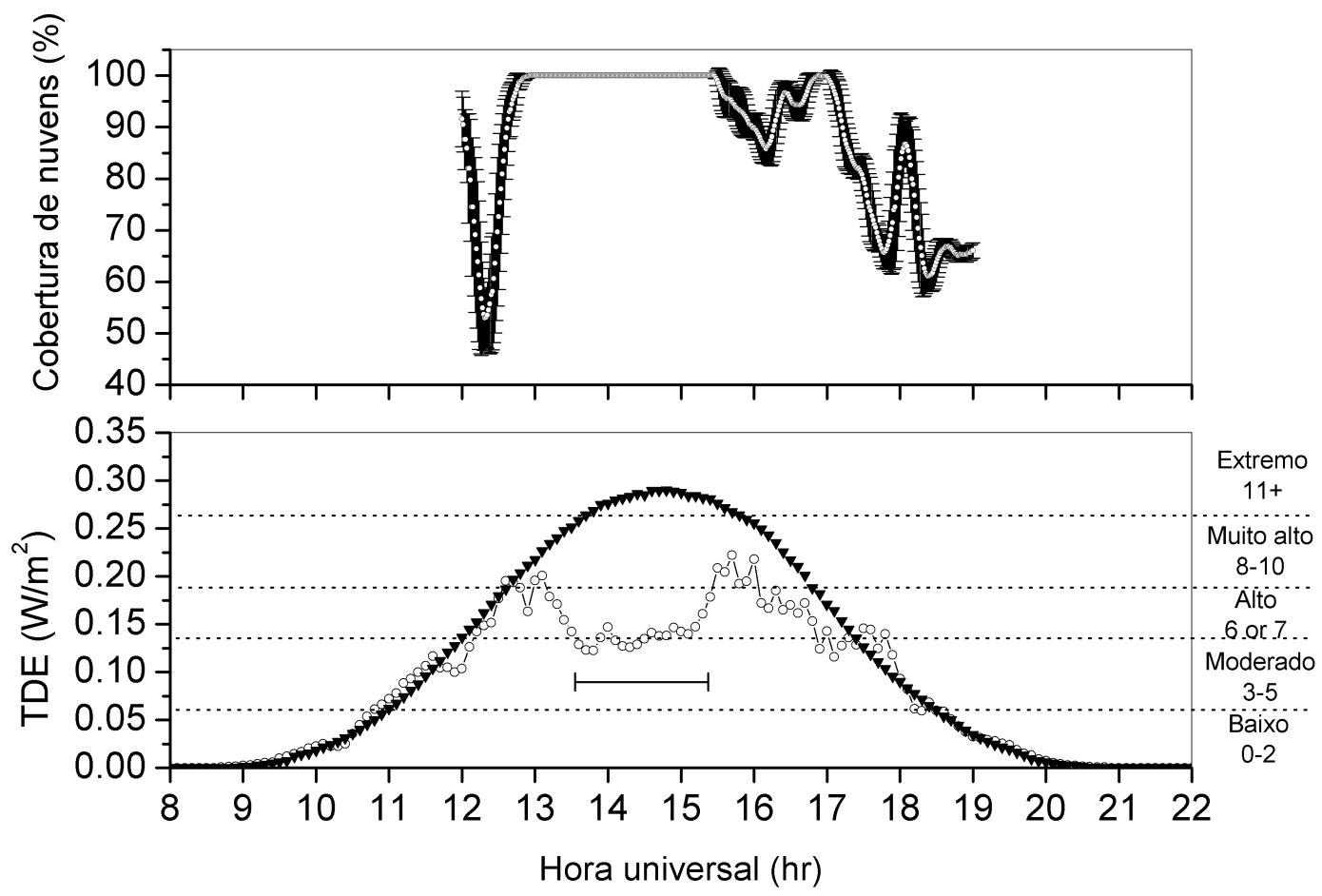

Figura 2 - (Superior) Cobertura de nuvens em BH no dia 21 de outubro de 2007 obtida a partir das imagens do TSI. Os valores são médias corridas em intervalos de 15 minutos tomados a cada 1 minuto e as incertezas iguais a 1 desvio padrão. (Inferior) Os círculos indicam a TDE medida no dia 294, enquanto os triângulos escuros indicam a TDE horizontal demarca o período em estudo.

2008). 0 maior valor dessa taxa foi de $0,222 \mathrm{~W} / \mathrm{m}^{2}$ correspondendo à faixa de IUV muito alto, enquanto que durante o período com $100 \%$ de As a TDE esteve basicamente na faixa de IUV alto. Para ambas as faixas a recomendação numa exposição é 0 uso de protetor solar além de óculos escuros, chapéu e roupas cobrindo braços e pernas, evitando prolongar-se sob o Sol além do estritamente necessário (WHO, 2002). Contudo, o público em geral não costuma ter disponível em tempo real a informação do IUV, além 
do fato de que o cenário meteorológico era muito convidativo para se estar ao ar livre com pouca roupa, sem chapéu e sem óculos escuros uma vez que não havia incidência direta de radiação solar. Por esse mesmo motivo, também não é improvável que as pessoas tenham se descuidado em relação ao uso da loção protetora solar (Benvenuto-Andrade et al., 2005; Szklo et al., 2007; Silva, 2008), ou mesmo abolido essa prática.

Naquelas faixas de IUV, pessoas com pele tipos I e II (WHO, 2002; Silva, 2008), ou seja, pele clara, perceberiam através do eritema que não tardaria a aparecer que a incidência de RUV, na verdade, estava elevada a despeito da cobertura de nuvens. Mas pessoas com tipos de pele mais escura provavelmente não desenvolveriam um eritema de forma perceptível tão prontamente, tendendo a se expor por mais tempo. 0 eritema por si só não é um dos efeitos mais graves da RUV, os demais malefícios dessa radiação são piores que ele e, em sua maioria, causados por doses acumuladas ao longo dos anos em situações de exposição como, por exemplo, a baseada na crença de que as nuvens em geral são uma boa proteção contra a RUV. Em cenários como 0 deste estudo de caso, as pessoas poderiam ser induzidas a permanecer ao ar livre por longos períodos sob o pressuposto de que a TDE estaria na faixa baixo do IUV quando, na verdade, ela está na faixa alto desse índice.

Pode-se simular com boa aproximação a incidência de TDE no dia 294 se esse tivesse sido um dia de céu sem nuvens através da correção pela coluna de ozônio da TDE de um dia próximo àquele onde realmente não houve cobertura de nuvens. Considerar dois dias próximos garante que a variação na distância SolTerra é pequena o bastante para ser negligenciada e de certa forma que as contribuições de aerossóis e gases traço na atenuação da RUV têm valores similares típicos para a estação. A correção pela coluna de ozônio pode ser feita utilizando a fórmula de potência (Madronich, 1993),

$$
T D E_{c o r}=T D E_{278}\left(\frac{N_{278}}{N_{294}}\right)^{F A R}
$$

onde $\mathrm{N}$ representa 0 montante da coluna de ozônio e FAR é 0 fator de amplificação relativo ao efeito biológico considerado, neste caso é 0 eritema e FAR $=1,1$. Os índices 278 e 294 referem-se aos dois dias de outubro considerados, isto é, dias $5 \mathrm{e}$ 21 , respectivamente. 0 primeiro foi um dia com período diurno sem nuvens cuja coluna de ozônio foi de 296,57 Unidades Dobson, enquanto no segundo a coluna foi de 262,42 UD. Os valores de coluna de ozônio específicos para BH foram obtidos no sítio do Ozone Monitoring Instrument (http://avdc.gsfc.nasa.gov/ index.php?site=1593048672\&id=28), que é um experimento vo- ando a bordo do satélite Aura da agência espacial norte americana com a missão, entre outras, de medir a coluna de ozônio na atmosfera terrestre (Schoeberl et al., 2004). Os valores de TDE do dia 278 corrigidas $\left(T_{D E}\right.$ cor $)$ correspondem à curva de triângulos escuros na Figura 2 e representam a incidência de TDE no dia 294 se este tivesse sido um dia de céu sem nuvens. Como o dia 294 foi de nebulosidade elevada e variável (veja a parte superior da Fig. 2), é possível que o fenômeno de amplificação de RUV por reflexão em nuvens (Piacentini et al., 2003) tenha ocorrido algumas vezes ao longo do dia resultando em valores de TDE superiores aos valores corrigidos TDE Fig. 2, especialmente a parte da tarde). A diferença entre as duas curvas durante 0 evento de $100 \%$ de cobertura de As fornece com boa aproximação a atenuação da RUV pela camada de nuvens. Assim, a atenuação variou de $37 \%$ a $56 \%(0,102$ a $0,158 \mathrm{~W} / \mathrm{m}^{2}$, lembrando que cada $0,025 \mathrm{~W} / \mathrm{m}^{2}$ de TDE equivale a uma unidade de IUV) do correspondente valor de TDE sar desse percentual elevado de atenuação, a incidência de TDE durante a cobertura de As ainda atingiu a faixa alto de IUV que, além disso, por quase 6 horas esteve nas faixas alto e muito alto. A DE durante a 1 hora e 54 minutos de cobertura de As foi de $1001 \mathrm{~J} / \mathrm{m}^{2}$, o que equivale a $24 \%$ da dose total naquele dia.

Esses resultados não podem ser generalizados para toda nuvem As, mas servem de orientação e advertência para o comportamento a ser tomado em atividades ao ar livre em dias nublados. Num futuro próximo será publicada uma análise da TDE incidente para diversos casos de céu completamente encoberto à hora do almoço, que é o período onde incide cerca de $50 \%$ da DE diária (Silva \& Gabrich, 2007).

\section{CONCLUSÕES}

Neste artigo, foi mostrado que uma espessa camada de As encobrindo todo 0 céu não é suficiente para diminuir a TDE incidente a um nível seguro em termos de efeito de eritema (IUV não superior a 2) e, portanto, também não o é em termos dos outros malefícios da RUV para os quais a dose é acumulativa. Assim, no dia 21 de outubro de 2007, na primavera de BH, o IUV esteve por cerca de 6 horas nas faixas alto e muito alto a despeito de um céu encoberto na maior parte do tempo. Embora houvesse uma espessa camada de As além possivelmente de outros tipos de nuvens, a DE incidente nesse período foi de aproximadamente $1 / 4\left(1001 \mathrm{~J} / \mathrm{m}^{2}\right)$ do montante para aquele dia.

Uma combinação de aspectos meteorológicos (temperatura amena, sem chuva e sem incidência direta de radiação solar) favorecendo o lazer ao ar livre num domingo de primavera, adicio- 
nada a, infelizmente, uma ainda grande desinformação por parte da população em geral sobre o tema exposição solar, formam uma mistura que pode levar muitas pessoas a uma exposição excessiva a RUV em dias nublados como aquele. Portanto, os resultados deste estudo de caso podem substanciar de forma quantitativa as campanhas de conscientização/informação do público sobre hábitos saudáveis de exposição ao Sol em atividades ao ar livre em dias nublados.

\section{AGRADECIMENTOS}

À Fundação de Amparo à Pesquisa do Estado de Minas Gerais (FAPEMIG, projeto CRA 025/03) e ao Conselho Nacional de Desenvolvimento Científico e Tecnológico (CNPq, projeto 471159/04-2).

\section{REFERÊNCIAS}

ADAM MEN \& SHAZLY SME. 2007. Attenuation of UV-B radiation in the atmosphere: Clouds effect, at Qena (Egypt). Atmos. Environ., 41: 4856-4864.

BENVENUTO-ANDRADE C, ZEN B, FONSECA G, DE VILLA D \& CESTARI T. 2005. Sun exposure and sun protection habits among high-school adolescents in Porto Alegre, Brazil. Photochem. Photobiol., 81: 630635 .

BORKOWSKI J, CHAI A-T, MO T \& GREEN AEO. 1977. Cloud effects on middle ultraviolet global radiation. Acta Geo. Polonica, 25: 287-301.

CALBÓ J, PAGÈS D \& GONZÁLEZ J-A. 2005. Empirical studies of cloud effects on UV radiation: A review. Rev. Geophys., 43, doi: 10.1029/ 2004RG000155.

CEDE A, BLUMTHALER M, LUCCINI E, PIACENTINI RD \& NUÑEZ L. 2002. Effects of clouds on erythemal and total irradiance as derived from data of the Argentine network. Geo. Res. Lett., 29, doi: 10.1029/ 2002GL015708.

CRAWFORD J, SHETTER RE, LEFER B, CANTRELL C, JUNKERMANN W, MADRONICH S \& CALVERT J. 2003. Cloud impacts on UV spectral actinic flux observed during the International Photolysis Frequency Measurement and Model Intercomparison (IPMMI). J. Geophys. Res., 108, doi: 10.1029/2002JD002731.

GODAR DE. 2005. UV doses worldwide. Photochem. Photobiol., 81: 736-749.

HERMAN JR, CELARIER E \& LARKO D. 2001a. UV $380 \mathrm{~nm}$ reflectivity of the Earth's surface, clouds and aerosols. J. Geophys. Res., 106: 53355351.

HERMAN JR, LARKO D, CELARIER E \& ZIEMKE J. 2001b. Changes in the Earth's reflectivity from the surface, clouds, and aerosols. J. Geophys. Res., 106: 5353-5368.
HOLLE RL \& MacKAY AS. 1975. Tropical cloudiness from all-sky cameras on Barbados and adjacent Atlantic ocean. J. App. Meteor., 14: 1437-1450.

HÜLSEN G \& GRÖBNER J. 2007. Characterization and calibration of ultraviolet broadband radiometers measuring erythemally weighted irradiance. Appl. Opt., 46: 5877-5886.

INCA. Instituto Nacional de Câncer. 2007. Estimativa 2008: incidência de câncer no Brasil. Rio de Janeiro: INCA. 94 p.

KRZYSCIN JW, JAROSLAWSKI J \& SOBOLEWSKI OS. 2003. Effects of clouds on the surface erythemal UV-B irradiance at northern midlatitudes: estimation from the observations taken at Belsk, Poland (1999-2001). J. Atmos. Sol.-Terr. Phys., 65: 457-467.

KULLAVANIJAYA P \& LIM HW. 2005. Photoprotection. J. Am. Acad. Dermatol., 52: 937-958.

KYLLING A, ALBOLD A \& SECKMEYER G. 1997. Transmittance of a cloud is wavelength-dependent in the UV-range: Physical interpretation. Geophys. Res. Lett., 24: 397-400.

LONG CN, SABBURG JM, CALBÓ J \& PAGÈS D. 2006. Retrieving cloud characteristics from ground-based daytime color all-sky images. J. Atmos. Oceanic Technol., 23: 633-652.

LOVENGREEN C, FUENZALIDA H \& VIDELA L. 2005. On the spectral dependency of UV radiation enhancements due to clouds in Valdivia, Chile (39.8S). J. Geophys. Res., 110, doi: 10.1029/2004JD005372.

MADRONICH S. 1993. UV radiation in the Natural and perturbed atmosphere. In: TEVINI M (Ed.). UV-B radiation and ozone depletion - Effects on humans, animals, plants, microorganisms, and material. Lewis Publishers, Boca Raton, $248 \mathrm{p}$.

MAYER B, KYLLING A, MADRONICH S \& SECKMEYER G. 1998. Enhanced absorption of UV radiation due to multiple scattering in clouds: Experimental evidence and theoretical explanation. J. Geophys. Res., 103: 31241-31254.

McKENZIE RL, AUCAMP PJ, BAIS AF, BJÖRN LO \& ILYAS M. 2007. Changes in biologically-active ultraviolet radiation reaching the Earth's surface. Photochem. Photobiol. Sci., 6: 218-231.

McKINLAY AF \& DIFFEY BL. 1987. A reference action spectrum for ultraviolet induced erythema in human skin. CIE J., 6: 17-22.

PETERS BSM, SANTOS LC, FISBERG M, WOOD RJ \& MARTINI LA. 2009. Prevalence of vitamin $D$ insufficiency in Brazilian adolescents. Ann. Nutr. Metab., 54: 15-21.

PFISTER G, McKENZIE RL, LILEY JB \& THOMAS A. 2003. Cloud based on all-sky imaging and its impact on surface solar irradiance. Journal of Applied Meteorology, 42: 1421-1434.

PIACENTINI RD, CEDE A \& BÁRCENA H. 2003. Extreme solar total and UV irradiances due to cloud effect measured near the summer solstice at 
the high-altitude desertic plateau Puna of Atacama (Argentina). J. Atmos. Sol.-Terr. Phys., 65: 727-731.

SABBURG JM \& PARISI AV. 2006. Spectral dependency of cloud enhanced UV irradiance. Atmos. Res., 81: 206-214.

SCHAFER JS, SAXENA VK, WENNY BN, BARNARD W \& DE LUISI JJ. 1996. Observed influence of clouds on ultraviolet-B radiation. Geophys. Res. Lett., 23: 2625-2658.

SCHOEBERL MR, DOUGLAS AR, HILSENRATH E, BHARTIA PK, BARNETT J, GILLE J, BEER R, GUNSON M, WATERS J, LEVELT PF \& DECOLA P. 2004. Earth observing system missions benefit atmospheric research. EOS, 85: 177-184.

SECKMEYER G, ERB R \& ALBOLD A. 1996. Transmittance of a cloud is wavelength-dependent in the UV-range. Geo. Res. Lett., 23: 2753-2755.

SILVA AA. 2007. A quarter century of TOMS total column ozone measurements over Brazil. J. Atmos. Sol.-Terr. Phys., 69: 1447-1458.

SILVA AA. 2008. Medidas de radiação solar ultravioleta em Belo Horizonte e saúde pública. Revista Brasileira de Geofísica, 26: 417-425.
SILVA AA. 2009. Daily distribution of UV-Index in Belo Horizonte (Brazil) and the shadow rule. Revista Brasileira de Geofísica, 27: 313-322.

SILVA AA \& GABRICH LLP. 2007. Seasonal Erythemal UV Doses in Belo Horizonte, Brazil. Photochem. Photobiol., 83: 1197-1204.

SOUZA SRP, FISCHER FM \& SOUZA JMP. 2004. Bronzeamento e risco de melanoma cutâneo: revisão da literatura. Revista de Saúde Pública, 38: 588-598.

SOUZA-ECHER MP, MARTINS FR \& PEREIRA EB. 2006. A importância dos dados de cobertura de nuvens e de sua variabilidade: Metodologias para aquisição de dados. Revista Bras. Ens. Física, 28: 341-352.

SZKLO AS, ALMEIDA LM, FIGUEIREDO V, LOZANA JA, MENDONÇA GAS, MOURA L \& SZKLO M. 2007. Comportamento relativo à exposição e proteção solar na população de 15 anos ou mais de 15 capitais brasileiras e Distrito Federal, 2002-2003. Cad. Saúde Pública, 23: 823-834.

WMO. World Meteorological Organization. 1956. International cloud Atlas. Paris: WMO. 155 p.

WHO. World Health Organization. 2002. Global Solar UV-Index: a practical guide. Geneva: WHO. 28 p.

\section{NOTA SOBRE O AUTOR}

Abel A. Silva. Doutor em Geofísica Espacial pelo Instituto Nacional de Pesquisas Espaciais. Atualmente é pesquisador do Instituto de Estudos Avançados (IEAv) e professor adjunto da Pontifícia Universidade Católica de Minas Gerais (PUC Minas) onde coordena o Laboratório de Luz Ultravioleta (LLUV, www.dfa.pucminas.br/PUV/ index.html). Atua nas áreas de Geofísica Espacial e Nuclear nos seguintes temas: instrumentação para medidas de radiação; radiações ionizantes e não-ionizantes, radiação atmosférica (radônio e seus descendentes, radiação solar ultravioleta); parâmetros geofísicos atmosféricos (ozônio, nuvens e aerossóis). 